\title{
A far-red fluorescent chemogenetic reporter for in vivo molecular imaging
}

Chenge Li ${ }^{1, \#, \mp}$, Alison G. Tebo ${ }^{1,2, \#}$, Marion Thauvin ${ }^{3,4, \#}$, Marie-Aude Plamont ${ }^{1}$, Michel Volovitch $^{3,5}$, Xavier Morin ${ }^{6}$, Sophie Vriz ${ }^{3,7}$, Arnaud Gautier ${ }^{1,2,8, *}$

1 PASTEUR, Department of Chemistry, École Normale Supérieure, Université PSL, Sorbonne Université, CNRS, 75005 Paris, France.

2 Sorbonne Université, École Normale Supérieure, Université PSL, CNRS, Laboratoire des biomolécules, LBM, 75005 Paris, France

${ }^{3}$ Center for Interdisciplinary Research in Biology (CIRB), Collège de France, CNRS, INSERM, Université PSL, Paris, France

${ }^{4}$ Sorbonne Université, Paris, France

${ }^{5}$ École Normale Supérieure, Université PSL, Department of biology, Paris, France ${ }^{6}$ Institut de Biologie de I'ENS (IBENS), École Normale Supérieure, CNRS, INSERM, Université PSL, 75005 Paris, France

${ }^{7}$ Université de Paris, Faculty of Science - 75006 Paris, France

${ }^{8}$ Institut Universitaire de France

\# equal contributions

*Current address: (a) Department of Obstetrics and Gynecology, Ren Ji Hospital, School of Medicine, Shanghai Jiao Tong University; (b) State Key Laboratory of Oncogenes and Related Genes, Shanghai Cancer Institute, Ren Ji Hospital, School of Medicine, Shanghai Jiao Tong University

Correspondence should be addressed to: arnaud.gautier@sorbonne-universite.fr 


\section{Abstract}

Far-red emitting fluorescent labels are highly desirable for spectral multiplexing and deep tissue imaging. Here, we describe the generation of frFAST (far-red Fluorescence Activating and absorption Shifting Tag), a 14-kDa monomeric protein that forms a bright far-red fluorescent assembly with (4-hydroxy-3-methoxy-phenyl)allylidene rhodanine (HPAR-3OM). As HPAR-3OM is essentially non-fluorescent in solution and in cells, frFAST can be imaged with high contrast in presence of free HPAR-3OM, which allowed the rapid and efficient imaging of frFAST fusions in live cells, zebrafish embryo/larvae and chicken embryo. Beyond enabling genetic encoding of far-red fluorescence, frFAST allowed the design of a far-red chemogenetic reporter of protein-protein interactions, demonstrating its great potential for the design of innovative far-red emitting biosensors. 


\section{INTRODUCTION}

The high demand for far-red fluorescent labels has emerged from the need for new colors as the growing use of optical biosensors and optogenetic tools obstruct the spectral window available for biological imaging. Moreover, far-red fluorescent labels allow imaging deeper in tissues as the autofluorescence, light scattering and absorbance by endogenous molecules is reduced in the far-red region. The first far-red fluorescent proteins were engineered from phytochromes and phycobiliproteins ${ }^{1-3}$ 4-6. These engineered fluorescent proteins covalently bind biliverdin, which fluoresces in the far-red region once attached to the protein. Far-red fluorescent proteins were successfully imaged in cells and animals; however, the availability of intracellular biliverdin and the rather slow rate of chromophore attachment (half-time of maturation > $100 \mathrm{~min}$ ) was shown to affect the efficiency of labeling ${ }^{7}$.

Chemogenetic fluorescent reporters based on self-labeling tags and fluorogenactivating proteins have been proposed as alternative to encode far-red fluorescence and label proteins in cells and multicellular organisms applying synthetic fluorogenic dyes exogenously ${ }^{8-11}$. Here, we describe frFAST, a small monomeric protein tag of 14 $\mathrm{kDa}$ that forms a far-red fluorescent assembly with (4-hydroxy-3-methoxyphenyl)allylidene rhodanine (HPAR-3OM) (Figure 1a) and enables the observation of fusion proteins in live cells and organisms. frFAST is a variant of FAST (FluorescenceActivating and absorption-Shifting Tag), a monomeric protein tag engineered from the apo photoactive yellow protein (PYP) from Halorhodospira halophila. In its original form, FAST forms with an exquisite selectivity fluorescent complexes with derivatives of 4hydroxybenzylidene rhodanine (HBR $)^{12,13}$ (Supplementary Figure 1), which are 
otherwise non-fluorescent chromophores. This strong fluorogenic effect allows the imaging of FAST-tagged proteins in cells and multicellular organisms with high contrast. Labeling of FAST-tagged proteins in cells takes place within a few seconds and can be reversed by simple washing, if needed. With a size of $14 \mathrm{kDa}, \mathrm{FAST}$ is one of the smallest genetically encoded tags, limiting the risk of dysfunctional fusions and minimizing its genetic footprint. In addition, as it does not require molecular oxygen to be fluorescent, FAST was shown to be an attractive alternative to classical fluorescent proteins for imaging biological phenomena in anaerobic systems ${ }^{14,15}$. Introduction of a single mutation (V107I) and/or tandemerization was furthermore shown to be an efficient way to improved brightness ${ }^{16}$. Imaging of proteins below the diffraction limit using super-resolution imaging by radial fluctuations ${ }^{17}$ or single-molecule localization microscopy (SMLM) ${ }^{18}$ could moreover be achieved thanks to the ability to label only a subset of FAST-tagged proteins by adjusting the chromophore concentration. Because of its modular nature, FAST allowed also the design of a $\mathrm{Ca}^{2+}$ biosensor $^{19}$ and a fluorescent split reporter, splitFAST, with rapid and reversible complementation for the detection of transient protein-protein interactions ${ }^{20}$.

In terms of spectral properties, the emission peak of FAST could be tuned from $540 \mathrm{~nm}$ to $600 \mathrm{~nm}$ by changing the substituents on the aromatic ring of the HBR scaffold $^{13}$ (Supplementary Figure 1). To shift the spectral properties to the far-red, we used a coordinated strategy of fluorogen engineering and protein engineering to develop frFAST, a FAST variant able to recognize HPAR-3OM. Similarly to HBR derivatives, HPAR-30M exhibits essentially undetectable fluorescence in solution or in cells, but displays red-shifted spectral properties because of an extra double bond 
between the phenol and the rhodanine moieties which elongates the $\pi$-electron conjugation. We demonstrated the efficiency of frFAST for multicolor imaging in cells and chicken embryo, and for in vivo imaging. frFAST was furthermore used to design a far-red fluorescent reporter for the detection of rapid and transient protein-protein interactions.

\section{RESULTS}

\section{Engineering and characterization of frFAST}

HBR analogs are non-fluorescent in solution, but they fluoresce strongly when immobilized within FAST due to inaccessible radiationless decay channels. Stabilization of their phenolate state within FAST red-shifts their absorption. This spectral change enables one to selectively excite FAST without exciting free chromophores protonated at physiological $\mathrm{pH}$, further increasing the fluorogenic effect. It is assumed that FAST stabilized the phenolate state of HBR analogs via the same network of hydrogen bonds that stabilizes the phenolate state of the hydroxycinnamoyl chromophore within the parental PYP ${ }^{12,16}$.

In order to push the spectral properties further to the red edge of the visible spectrum, we added a double bond between the electron-donating phenol and the electron-withdrawing rhodanine heterocycle. The 4-hydroxyphenylallylidene rhodanine (HPAR) was previously shown to display red-shifted absorption and emission because of extended $\pi$-electron conjugation ${ }^{21}$. We generated binders able to activate the fluorescence of HPAR using a library of $10^{6}$ variants of FAST displayed on yeast cells.

This library was incubated with HPAR and screened by fluorescence-activating cell 
sorting. Iterative rounds of sorting allowed us to identify a variant with the mutations D71V and P73T that formed a fluorescent complex with HPAR, characterized by 500 $\mathrm{nm} / 635 \mathrm{~nm}$ absorption-emission peaks and a fluorescence quantum yield $\phi=8 \%$.

We tested the properties of FAST ${ }^{\mathrm{D} 71 \mathrm{~V}, \mathrm{P} 73 \mathrm{~T}}$ with various HPAR analogs, and discovered that HPAR-3OM, which bears an additional methoxy group on the aromatic ring, formed a complex with FAST ${ }^{\mathrm{D} 71 \mathrm{~V}, \mathrm{P} 73 \mathrm{~T}}$ displaying further red-shifted absorptionemission peaks (528 nm / $663 \mathrm{~nm}$ ) and a higher fluorescence quantum yield, $\phi=13 \%$. This was consistent with our previous observations that modifications to the electrondonating phenol moiety could redshift the absorption and emission of this class of pushpull molecules ${ }^{13}$. We thus used directed evolution to further improve the properties of FAST $^{\mathrm{D} 71 \mathrm{~V}, \mathrm{P73T}}$ with HPAR-3OM. A library of $6 \times 10^{7}$ variants was prepared by random mutagenesis and displayed on yeast cells. Iterative rounds of sorting in presence of HPAR-3OM allowed us to select an improved variant, which was further refined by introduction of the mutation V107I previously described to increase FAST brightness ${ }^{16}$. The resulting variant, possessing the mutations F62L, D71V, P73S, E74G and V107I relative to FAST, binds HPAR-3OM tightly with a $K_{D}$ of $1 \mu \mathrm{M}$ (Supplementary Figure 2), and strongly activates its far-red fluorescence (Figure 1b), forming a fluorescent complex with $555 \mathrm{~nm} / 670 \mathrm{~nm}$ absorption-emission peaks, a fluorescence quantum yield $\phi=21 \%$, and a molar absorptivity $\varepsilon=45,000 \mathrm{M}^{-1} \cdot \mathrm{cm}^{-1}$. HPAR-3OM undergoes a $130 \mathrm{~nm}$ red shift in absorption upon binding, in accordance with a change of ionization state from protonated in solution to deprotonated when bound (Figure $\mathbf{1 b}$ left), ensuring that unbound HPAR-3OM is fully invisible (because it does not absorb) at the wavelength used for exciting the complex. The width of the absorption band allows 
efficient excitation with the $543 \mathrm{~nm}, 594 \mathrm{~nm}$ and $633 \mathrm{~nm}$ lines of helium-neon lasers or the $561 \mathrm{~nm}$ line of yellow diode lasers. Because of the far-red fluorescence of the complex and the red-shift in absorption undergone by HPAR-3OM upon complexation, this improved variant was designated far-red fluorescence activating and absorption shifting tag (frFAST). With a large Stokes shift $(=115 \mathrm{~nm})$, frFAST is singular among the far-red fluorescent proteins, which usually display narrower Stokes shifts, offering thus new possibilities for biological imaging. Note that frFAST conserves the ability to bind HBR analogs, although with lower performances than FAST variants (Supplementary Table 1).

\section{Imaging frFAST in live cells}

We next asked whether frFAST could be used to encode far-red fluorescence in living cells. We first verified that HPAR-3OM had no deleterious effects on cells at the concentrations used for labeling. We exposed HeLa cells to $10 \mu \mathrm{M}$ HPAR-3OM for 24 hours. No effects on cell health and viability were observed (Supplementary Figure 3). We next expressed frFAST in HeLa and U2OS cells, and treated cells with $10 \mu \mathrm{M}$ HPAR-3OM. Robust far-red fluorescence was seen in transfected cells after 15 seconds of incubation. Furthermore, expression of frFAST was homogenous and no intracellular aggregates were observed (Figure 1c).

We then verified that frFAST could be used as a tag for labeling intracellular proteins. We expressed frFAST protein fusions with the histone $\mathrm{H} 2 \mathrm{~B}$ (H2B-frFAST), the mitochondrial targeting sequence from the subunit VIII of human cytochrome c oxidase (Mito-frFAST), and the microtubule-associated protein (MAP) 4 (MAP4-frFAST) in 
HeLa and U2OS cells. Treatment with HPAR-3OM led to far-red fluorescence in the expected localizations, demonstrating that frFAST fusions were functional and exhibited correct behavior (Figure 1c).

We next tested the photostability of frFAST:HPAR-3OM. Long-term imaging of HeLa cells expressing frFAST fused to H2B allowed us to show that frFAST displayed high photostability (Supplementary Figure 4). In direct comparison with smURFP5 and iRFP670 3 - two dimeric far-red fluorescent proteins having emission properties comparable to frFAST - frFAST appeared a bit less photostable than iRFP670 but more than smURFP (Supplementary Figure 4), which was previously reported to be more photostable than most monomeric far-red fluorescent proteins ${ }^{6}$.

We next evaluated the use of frFAST for multi-color imaging. We imaged live HPAR-3OM-treated mammalian cells co-expressing frFAST, EGFP and mCherry fusions using a confocal microscope with adjustable emission bandwidth (Figure 1d). All fusions showed the expected localizations and clear spectral separation of their fluorescence signals, which allowed us to perform three-color imaging in live cells. This set of experiments showed that frFAST could be used as an additional reporter for highly multiplexed imaging.

\section{Imaging protein-protein interactions in live cells}

One of the advantages of the FAST system is that its reversible interaction with HBR analogs permitted the development of splitFAST, a split fluorescent reporter with reversible complementation for the visualization of dynamic protein-protein interactions ${ }^{20}$. We thus examined whether this property was retained by frFAST, despite 
the changes to the protein that allow it to recognize this class of larger fluorogen. Two complementary fragments were generated by splitting frFAST in its last loop between Ser 114 and Gly 115 (Figure 2a). To evaluate the ability of the two fragments to complement when fused to two interacting proteins, C-frFAST (fragment 115-125) and $\mathrm{N}$-frFAST (fragment 1-114) were fused respectively to the FK506-binding protein (FKBP) and the FKBP-rapamycin-binding domain of mammalian target of rapamycin (FRB), two proteins that interact together in the presence of rapamycin. HEK 293T cells co-expressing FRB-N-frFAST and FKBP-C-frFAST were treated with $10 \mu \mathrm{M}$ HPAR3OM. Induction of FRB-FKBP dimerization by addition of rapamycin led to an increase of the far-red fluorescence, in accordance with an interaction-dependent complementation of the two split fragments (Figure $\mathbf{2 b - d}$ ). The median fluorescence fold increase was 7-fold (Figure 2c). Time-lapse imaging showed that complementation occurred within a few minutes after addition of rapamycin (Figure $\mathbf{2 b} \mathbf{b}$ ), in agreement with the rapid formation of the FRB-FKBP-rapamycin complex.

We next tested the reversibility of split-frFAST using the ability of rapamycin to dissociate an AP1510-induced FKBP homodimer. We co-expressed FKBP-N-frFAST and FKBP-C-frFAST in HEK 293T cells. Cells were pre-treated with AP1510 for $2 \mathrm{~h}$ to form the FKBP homodimer and $10 \mu \mathrm{M}$ HPAR-3OM was added to visualize the complemented split-frFAST. The addition of rapamycin led to a median fluorescence fold decrease of 5-fold, in agreement with a disassembly of split-frFAST concomitant with the dissociation of the FKBP homodimer (Figure $\mathbf{2 e - g}$ ). Time-lapse imaging showed that complementation was reversed within a few minutes (Figure $\mathbf{2 e , g}$ ), in agreement with the rapid disassembly of FKBP homodimer. 
Overall, this set of experiments suggested that split-frFAST could be used to monitor protein complex formation and dissociation with high spatial and temporal resolution.

\section{Imaging frFAST in live zebrafish embryos and larvae}

The use of far-red fluorescent tags is particularly important when working with multicellular organisms, due to the lower autofluorescence and the better light penetration in tissues at longer wavelengths. We thus examined the performance of frFAST in multicellular organisms. As model, we first used zebrafish embryo and larvae. We initially tested the toxicity of HPAR-3OM and frFAST. Zebrafish embryos were incubated with $5 \mu \mathrm{M}$ HPAR-3OM during 1 hour at 50\% epiboly, or overnight from $50 \%$ epiboly to $24 \mathrm{hpf}$. In both cases, no significant deleterious effects were observed at 48 hpf (Supplementary Figure 5a). The effect of expressing an frFAST fusion was tested by injecting mRNA coding for H2B-frFAST in zebrafish embryos at the one-cell stage. Embryos developed correctly and no deleterious effects were observed at $24 \mathrm{hpf}$ (Supplementary Figure 5b). As a comparison, we observed that the expression of $\mathrm{H} 2 \mathrm{~B}$ fused to smURFP was highly toxic, and led to $50 \%$ dead embryos and $50 \%$ embryos with strong axis defects at $24 \mathrm{hpf}$.

We then evaluated the labeling efficiency in zebrafish embryos. We injected mRNA coding for H2B-frFAST in zebrafish embryo at the one-cell stage, together with an mRNA encoding H2B-EGFP to account for injection efficiency. Embryos were imaged after treatment with $5 \mu \mathrm{M}$ HPAR-3OM for $30 \mathrm{~min}$. We observed that expression of H2B-frFAST was efficiently detected during gastrulation (Figure 3a,b) and at 24 hpf 
(Figure 3c,d). Similar experiments using H2B fused to the far-red fluorescent protein iRFP713² revealed that iRFP713 could only be detected at $24 \mathrm{hpf}$ but not during gastrulation (Figure 3e-h). The absence of iRFP713 signal during gastrulation might be due to the slow incorporation of biliverdin and/or the lack of biliverdin at this development stage. Overall, these results demonstrated that frFAST could be an efficient far-red fluorescent tag for imaging proteins in zebrafish at various stages of embryogenesis, even early ones.

We next tested if the far-red fluorescence of frFAST could be detected in internal organs in zebrafish larvae. We established a transgenic line of zebrafish with expression of frFAST fused to LifeAct, a peptide binding filamentous actin, under the control of the cmlc2 promoter, known to be sufficient to drive myocardium-specific expression (Figure 3i). Labeling with HPAR-3OM and imaging with a high speedspinning disk confocal microscope allowed us to observe the myocardium in beating heart in 3 dpf larvae (Figure 3j and Supplementary Movie 1). This experiment showed that frFAST fusion could be efficiently labeled and detected in internal organs in live zebrafish larvae.

\section{Imaging frFAST in chicken embryos}

To verify that frFAST could be used in other multicellular organisms, we used chicken embryo as a second model. Plasmids encoding frFAST or miRFP670nano ${ }^{6}$ (fused to H2B) under the control of the CAGGS promoter ${ }^{22}$ were each electroporated in one of each side of the neural tube in ovo, at embryonic day 2 (E2, HH stage 13-14) (Figure 4a,b). An EGFP reporter (fused to a membrane localization signal) was co- 
injected with each construct to monitor electroporation efficiency. $24 \mathrm{~h}$ later, embryos with homogeneous bilateral EGFP expression in the neural tube were dissected and imaged in absence and in presence of HPAR-3OM (Figure 4c). Only cells expressing frFAST lighted up upon addition of HPAR-3OM, demonstrating the selective labeling of frFAST in chicken embryos. In addition, cells located in the side electroporated with the plasmid encoding frFAST showed higher fluorescence than cells located in the side electroporated with the plasmid encoding miRFP670nano when excited at $640 \mathrm{~nm}$, suggesting that frFAST outperformed miRFP670nano in terms of effective brightness in this context. Time-lapse imaging furthermore showed that maximal fluorescence was achieved 20-25 min after HPAR-3OM addition (Supplementary Movie 2), demonstrating that HPAR-3OM efficiently diffused across the embryo.

We next tested our ability to perform multicolor imaging in the chicken embryo. We electroporated plasmids encoding frFAST (fused to H2B) together with EGFP (fused to centrin2) and $\mathrm{mKO} 2$ (fused to a membrane localization signal) in the neural tube as previously (Figure 4d). En-face time-lapse imaging of the neuroepithelium with a spinning-disk confocal microscope allowed us to visualize the three proteins (Figure 4f) and follow their dynamics during cell division (Figure $\mathbf{4 g}, \mathbf{h}$ ). This set of experiments allowed us to further validate the use of frFAST for multicolor imaging of dynamic processes with a subcellular resolution in multicellular organisms.

\section{DISCUSSION}

A coordinated strategy of protein engineering and fluorogen engineering allowed us to generate a small far-red fluorescent chemogenetic reporter named frFAST, which forms 
a bright far-red fluorescent complex with the fluorogenic chromophore HPAR-3OM.

HPAR-3OM is essentially non-fluorescent in solution and in cells, allowing one to image proteins fused to frFAST with high contrast in the presence of free fluorogenic ligand.

The high fluorogenicity and exquisite labeling specificity allowed the imaging of proteins not only in mammalian cells but also in multicellular systems such as zebrafish embryo/larvae and chicken embryo. With 555 nm / 670 nm absorption-emission peaks, frFAST is a far-red fluorescent reporter with a large Stokes shift, making it singular in the landscape of far-red fluorescent proteins, which usually display narrow Stokes shifts (Supplementary Table 2), expanding thus the fluorescence toolkit with new properties and possibilities. Its far-red emission enabled us to perform multicolor imaging both in cells and in multicellular systems.

In contrast with biliverdin-based far-red fluorescent proteins, which display slow fluorescence maturation (half-time $>100 \mathrm{~min}$ ) because of the need to covalently bind biliverdin, frFAST binds HPAR-3OM almost instantaneously. Combined with the high cellular uptake of HPAR-3OM, this allows the labeling to occur with only few tens of seconds in mammalian cells and few tens of minutes in zebrafish embryos/larvae and chicken embryos. As frFAST requires the addition of an exogenous fluorogen, its brightness can be maximized by adjusting the HPAR-3OM concentration to have almost $100 \%$ fluorescent complex, avoiding the problems encountered with far-red fluorescent proteins, in which effective brightness can be limited by the availability and accessibility of biliverdin ${ }^{7}$. frFAST could for instance be visualized during gastrulation, a development stage where iRFP713 was not detectable in zebrafish. The small size of frFAST is another advantage. Small monomeric tags are desirable as they interfere less with the 
labeled proteins, and have minimal genetic footprint. The toxicity we observed in zebrafish when using the far-red fluorescent protein smURFP illustrates how fluorescent proteins can affect the function of labeled proteins. With a molecular weight of less than $14 \mathrm{kDa}$, frFAST is smaller than the smallest monomeric far-red fluorescent protein, miRFP670nano ${ }^{6}$, a 17 kDa protein engineered from a cyanobacteriochrome. Finally, with a fluorescence quantum yield of $21 \%$ and a molar absorptivity of $45,000 \mathrm{M}^{-1} \mathrm{~cm}^{-1}$, the molecular brightness (i.e. the product of the fluorescence quantum and the molar absorptivity) of frFAST equals or exceeds that of most monomeric far-red fluorescent proteins (Supplementary Table S2).

As a very close structural and functional relative of the chemogenetic reporter FAST, frFAST displays the same advantages. The need to add HPAR-3OM to generate the far-red fluorescence allows the design of experimental protocols requiring ondemand labeling. In addition, as no molecular oxygen is required for generating the farred fluorescence, frFAST should enable the detection of fusion proteins in anaerobic conditions. Finally, as FAST, frFAST should be well suited as optical reporting module for the design of various biosensors. We demonstrated in this work that frFAST could be turned, using the design previously used for the generation of splitFAST ${ }^{20}$, into a far-red split fluorescent reporter with rapid and reversible complementation for the detection of protein-protein interactions with no additional engineering. We anticipate that frFAST could be used for the design of far-red fluorescent biosensors through allosteric coupling of frFAST (or circularly permuted versions) and analyte binding domains, in the same way FAST was previously used for the design of biosensors ${ }^{19}$. 


\section{NOTES}

The authors declare the following competing financial interest: A.G. is co-founder and hold equity in Twinkle Bioscience / The Twinkle Factory, a company commercializing the FAST technology.

\section{ACKNOWLEDGEMENT}

We thank K. D. Wittrup, for providing us with the pCTCON2 vector and the EBY100 yeast strain for the yeast display selection. We also thank the flow cytometry facility CISA (Cytométrie Imagerie Saint-Antoine) of UMS LUMIC at the Faculty of Medicine of Sorbonne Université, and, more particularly, Annie Munier for her assistance. This work has been supported by the European Research Council (ERC-2016-CoG-724705 FLUOSWITCH).

\section{SUPPORTING INFORMATION}

Supporting information contains the Supplementary Movies 1-2, the Supplementary Tables 1-2 and the Supplementary Figures 1-5. 


\section{MATERIALS AND METHODS}

\section{General}

Synthetic oligonucleotides used for cloning were purchased from Sigma Aldrich or Integrated DNA Technology. PCR reactions were performed with Q5 polymerase (New England Biolabs) in the buffer provided. PCR products were purified using QIAquick PCR purification kit (Qiagen). The products of restriction enzyme digests were purified by preparative gel electrophoresis followed by QIAquick gel extraction kit (Qiagen). Restriction endonucleases, T4 ligase, Phusion polymerase, Taq ligase, and Taq exonuclease were purchased from New England Biolabs and used with accompanying buffers and according to manufacturer protocols. Isothermal assemblies (Gibson assembly) were performed using homemade mix prepared according to previously described protocols ${ }^{23}$. Small-scale isolation of plasmid DNA was done using QIAprep miniprep kit (Qiagen) from $2 \mathrm{~mL}$ of overnight culture. Large-scale isolation of plasmid DNA was done using the QIAprep maxiprep kit (Qiagen) from $150 \mathrm{~mL}$ of overnight culture. All plasmid sequences were confirmed by Sanger sequencing with appropriate sequencing primers (GATC services - Eurofins Genomics).

\section{Sequences}

Sequence of frFAST (125 amino acids, MW = 13,788.7 Da) MEHVAFGSEDIENTLAKMDDGQLDGLAFGAIQLDGDGNILQYNAAEGDITGRDPKQVI GKNLFKDVAPGTVSSGFYGKFKEGVASGNLNTMFEWMIPTSRGPTKVKIHMKKALSG DSYWVFVKRV

DNA sequence coding for frFAST (375 bp) atggagcatgttgcctttggcagtgaggacatcgagaacactttggccaaaatggacgacggacaactggatgggttggc ctttggcgcaattcagctcgatggtgacgggaatatcctgcagtacaatgctgctgaaggagacatcacaggcagagatc 
ccaaacaggtgattgggaagaacttattcaaggatgttgcacctggaacggtttcctccgggttttacggcaaattcaagga aggcgtagcgtcagggaatctgaacaccatgttcgaatggatgataccgacaagcaggggaccaaccaaggtcaaga tacacatgaagaaagccctttccggtgacagctattgggtctttgtgaaacgggtg

Sequence of N-frFAST (114 amino acids, MW = 12,251.15 Da) MEHVAFGSEDIENTLAKMDDGQLDGLAFGAIQLDGDGNILQYNAAEGDITGRDPKQVI GKNLFKDVAPGTVSSGFYGKFKEGVASGNLNTMFEWMIPTSRGPTKVKIHMKKALS

DNA sequence coding for N-frFAST (342 bp)

atggagcatgttgcctttggcagtgaggacatcgagaacacttggccaaaatggacgacggacaactggatgggttggc ctttggcgcaattcagctcgatggtgacgggaatatcctgcagtacaatgctgctgaaggagacatcacaggcagagatc ccaaacaggtgattgggaagaacttattcaaggatgttgcacctggaacggtttcctccgggtttacggcaaattcaagga aggcgtagcgtcagggaatctgaacaccatgttcgaatggatgataccgacaagcaggggaccaaccaaggtcaaga tacacatgaagaaagccctttcc

Protein sequence of C-frFAST (11 amino acids, MW 1,355.6 Da) GDSYWVFVKRV

DNA sequence coding for C-frFAST (33 bp)

ggtgacagctattgggtcttgtgaaacgggtg

\section{Fluorogens}

The synthesis of HPAR-3OM was performed using commercially available reagents used as received. To a stirred solution of rhodanine (133 mg, $1.0 \mathrm{mmol})$ and 4-Hydroxy3-methoxycinnamaldehyde (178 $\mathrm{mg}, 1.0 \mathrm{mmol})$ in ethanol $(10 \mathrm{~mL})$ was added 4(dimethylamino)pyridine $(12 \mathrm{mg}, 0.1 \mathrm{mmol})$. The solution was stirred at reflux for $20 \mathrm{~h}$. Acetic acid was added to neutralize the solution. After cooling at $4^{\circ} \mathrm{C}$ overnight, the precipitate was filtered. The crude solid was washed with diethyl ether and dried. HPAR-3OM was obtained pure as a red powder (150 mg, 52\% yield). ${ }^{1} \mathrm{H}$ NMR (300 MHz, DMSO-d6, $\delta$ in ppm) $13.54(\mathrm{~s}, 1 \mathrm{H}), 9.69(\mathrm{~s}, 1 \mathrm{H}), 7.33(\mathrm{~m}, 2 \mathrm{H}), 7.23$ (d, J = 15 $\mathrm{Hz}, 1 \mathrm{H}), 7.08(\mathrm{~d}, J=8.1 \mathrm{~Hz}, 1 \mathrm{H}), 6.84(\mathrm{~m}, 2 \mathrm{H}), 3.84(\mathrm{~s}, 3 \mathrm{H}) .{ }^{13} \mathrm{C}$ NMR $(75 \mathrm{MHz}$, DMSOd6, $\delta$ in ppm) 194.7, 168.6, 151.6, 146.6, 133.9, 130.0(2C), 123.1, 122.3, 118.3, 
111.9(2C), 39.7. HRMS (ESI): $\mathrm{m} / \mathrm{z} 294.0253[\mathrm{M}+\mathrm{H}]^{+}$, calcd mass for $\left[\mathrm{C}_{13} \mathrm{H}_{12} \mathrm{NO}_{3} \mathrm{~S}_{2}\right]^{+}$:

294.0259. ${ }^{1} \mathrm{H}$ and ${ }^{13} \mathrm{C}$ NMR spectra were recorded at room temperature on a Bruker AM 300 spectrometer. Mass spectrometry analysis was performed by the Institut de Chimie Organique et Analytique de l'Université d'Orléans.

The preparation of HPAR (4-hydroxy-phenylallylidene rhodanine) was previously described $^{21}$. The preparation of HMBR, HBR-3,5DM, HBR-3OM, HBR-3,5DOM was previously described ${ }^{12,13}$. HMBR, HBR-3,5DM, HBR-3OM, HBR-3,5DOM are commercially available from The Twinkle Factory (the-twinkle-factory.com) under the name ${ }^{\mathrm{TF}} \mathrm{Lime},{ }^{\mathrm{TF}} \mathrm{Amber},{ }^{\mathrm{TF}} \mathrm{C}$ itrus and ${ }^{\mathrm{TF}} \mathrm{C}$.ral, respectively.

\section{Directed evolution}

Library construction. The random mutagenesis library of FAST variants used for the initial yeast display screen with HPAR was previously described ${ }^{24}$. For the library of FAST $^{\mathrm{D} 71 \mathrm{~V}, \mathrm{P73T}}$ variants, the gene of FAST ${ }^{\mathrm{D} 71 \mathrm{~V}, \mathrm{P} 73 \mathrm{~T}}$ was randomly mutagenized by errorprone PCR using the GeneMorph II Random Mutagenesis Kit (Agilent). The PCR product was digested with $\mathrm{Nhel}$ and $\mathrm{BamHI}$, and then ligated into the pCTCON2 vector using Nhel / BamHI restriction sites. Large-scale transformation into DH10B E. coli cells was performed by electroporation. The DNA was then purified and retransformed into EBY100 yeast strain using a large-scale high-efficiency transformation protocol ${ }^{25}$. The final library contains $6 \times 10^{7}$ clones.

FACS selection. The yeast library (about $1 \times 10^{9}$ cells) was grown overnight $\left(30^{\circ} \mathrm{C}, 280\right.$ $\mathrm{rpm})$ in $1 \mathrm{~L}$ of $\mathrm{SD}(20 \mathrm{~g} / \mathrm{L}$ dextrose, $6.7 \mathrm{~g} / \mathrm{L}$ yeast nitrogen base, $1.92 \mathrm{~g} / \mathrm{L}$ yeast synthetic dropout without tryptophane, $7.44 \mathrm{~g} / \mathrm{L} \mathrm{NaH} \mathrm{PO}_{4}$ and $10.2 \mathrm{~g} / \mathrm{L} \mathrm{Na} \mathrm{HPO}_{4}-7 \mathrm{H} 2 \mathrm{O}, 1 \%$ 
penicillin-streptomycin 10,000 U/mL). Yeast culture was then diluted to $\mathrm{OD}_{600} 1 \mathrm{in} 1 \mathrm{~L}$ of $\mathrm{SD}$ and grown $\left(30^{\circ} \mathrm{C}, 280 \mathrm{rpm}\right)$ until $\mathrm{OD}_{600} 2-5 . \mathrm{Next}, 5 \times 10^{9}$ cells yeast cells were then collected and grown for $36 \mathrm{~h}\left(23^{\circ} \mathrm{C}, 280 \mathrm{rpm}\right)$ in $1 \mathrm{~L} \mathrm{SG}(20 \mathrm{~g} / \mathrm{L}$ galactose, $2 \mathrm{~g} / \mathrm{L}$ dextrose, $6.7 \mathrm{~g} / \mathrm{L}$ yeast nitrogen base, $1.92 \mathrm{~g} / \mathrm{L}$ yeast synthetic dropout without tryptophane, $7.44 \mathrm{~g} / \mathrm{L} \mathrm{NaH} \mathrm{PO}_{4}$ and $10.2 \mathrm{~g} / \mathrm{L} \mathrm{Na}_{2} \mathrm{HPO}_{4}-7 \mathrm{H} 2 \mathrm{O}, 1 \%$ penicillinstreptomycin $10,000 \mathrm{U} / \mathrm{mL}) .5 \times 10^{8}$ induced cells were then pelleted by centrifugation $\left(25^{\circ} \mathrm{C}, 3 \mathrm{~min}, 2,500 \mathrm{~g}\right)$, washed with $10 \mathrm{~mL}$ DPBS-BSA (137 mM NaCl, $2.7 \mathrm{mM} \mathrm{KCl}, 4.3$ $\mathrm{mM} \mathrm{Na}_{2} \mathrm{HPO}_{4}, 1.4 \mathrm{mM} \mathrm{KH}_{2} \mathrm{PO}_{4}, 1 \mathrm{~g} / \mathrm{L}$ bovine serum albumin, $\mathrm{pH}$ 7.4). Cells were incubated for $30 \mathrm{~min}$ at room temperature in $200 \mu \mathrm{L}$ of $1 / 250$ primary antibody chicken anti-Myc lgY (Life Technologies) solution in DPBS-BSA. Cells were then washed with

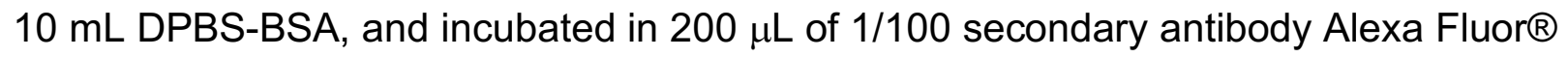
647-goat anti-chicken IgG (Life Technologies) solution in DPBS-BSA for 20 min on ice. Cells were then washed with DPBS. For the selection of HPAR binders using the library of FAST variants, cells were incubated in $10 \mathrm{~mL}$ DPBS supplemented with $10 \mu \mathrm{M}$ HPAR, and sorted on a MoFlo ${ }^{\mathrm{TM}}$ Astrios Cell Sorter (Beckman Coulter) equipped with a $488 \mathrm{~nm}$ and a $640 \mathrm{~nm}$ laser. The fluorescence of HPAR binders was detected using the following parameters: Ex 488 nm, Em $620 \pm 29$ nm. For the selection of HPAR-3OM binders using the library of FAST ${ }^{\mathrm{D} 71 \mathrm{~V}, \mathrm{P} 73 \mathrm{~T}}$ variants, cells were incubated in $10 \mathrm{~mL}$ DPBS supplemented with $5 \mu \mathrm{M}$ HPAR-3OM, and sorted on a MoFlo ${ }^{\mathrm{TM}}$ Astrios Cell Sorter (Beckman Coulter) equipped with a $561 \mathrm{~nm}$ laser and a $640 \mathrm{~nm}$ laser. The fluorescence of HPAR-3OM binders was detected using the following parameters: Ex $561 \mathrm{~nm}$, Em $664 \pm 11 \mathrm{~nm}$. For both selections, the sorted cells were collected in SD, grown overnight $\left(30^{\circ} \mathrm{C}, 240 \mathrm{rpm}\right)$ and spread on SD plates (SD supplemented with $182 \mathrm{~g} / \mathrm{L}$ sorbitol, 15 
$\mathrm{g} / \mathrm{L}$ agar). Plates were incubated for $60 \mathrm{~h}$ at $30^{\circ} \mathrm{C}$. The cell lawn was collected in SD supplemented with $30 \%$ glycerol, aliquoted and frozen or directly used in the next round. After FACS selection, clones were screened by flow cytometry and their DNA was isolated using a miniprep kit (Qiagen), transformed into $\mathrm{DH} 10 \mathrm{~B}$ and re-isolated for sequencing.

\section{Characterization of variants}

Cloning. Plasmids driving bacterial expression of the variants with an $\mathrm{N}$-terminal Histag under the control of a T7 promoter were constructed by inserting the encoding sequence between Nhel and Xhol restriction sites in the pET28a vector.

Expression. The plasmids were transformed in Rosetta(DE3)pLysS E. coli (New England Biolabs). Cells were grown at $37^{\circ} \mathrm{C}$ in Lysogeny Broth (LB) medium complemented with $50 \mu \mathrm{g} / \mathrm{ml}$ kanamycin and $34 \mu \mathrm{g} / \mathrm{ml}$ chloramphenicol to $\mathrm{OD}_{600 \mathrm{~nm}} 0.6$. Expression was induced at $37^{\circ} \mathrm{C}$ for $4 \mathrm{~h}$ by adding isopropyl $\beta-D-1$ -

thiogalactopyranoside (IPTG) to a final concentration of $1 \mathrm{mM}$. Cells were harvested by centrifugation $\left(4,300 \times \mathrm{g}\right.$ for $20 \mathrm{~min}$ at $\left.4^{\circ} \mathrm{C}\right)$ and frozen.

Purification protocol 1. The cell pellet was resuspended in lysis buffer (phosphate buffer $50 \mathrm{mM}, \mathrm{NaCl} 150 \mathrm{mM}, \mathrm{MgCl}_{2} 2.5 \mathrm{mM}$, protease inhibitor, DNase, $\mathrm{pH}$ 7.4) and sonicated ( 5 min at $20 \%$ of amplitude). The lysate was incubated for $2 \mathrm{~h}$ at $4{ }^{\circ} \mathrm{C}$ to allow DNA digestion by DNase. Cellular fragments were removed by centrifugation $\left(9,300 \times \mathrm{g}\right.$ for $1 \mathrm{~h}$ at $\left.4^{\circ} \mathrm{C}\right)$. The supernatant was incubated overnight at $4^{\circ} \mathrm{C}$ under gentle agitation with Ni-NTA agarose beads in phosphate buffered saline (PBS) (sodium phosphate $50 \mathrm{mM}, \mathrm{NaCl} 150 \mathrm{mM}, \mathrm{pH}$ 7.4) complemented with $10 \mathrm{mM}$ Imidazole. Beads 
were washed with 20 volumes of PBS containing $20 \mathrm{mM}$ Imidazole, and with 5 volumes of PBS complemented with $40 \mathrm{mM}$ Imidazole. His-tagged proteins were eluted with 5 volumes of PBS complemented with $0.5 \mathrm{M}$ Imidazole, followed by dialysis with PBS.

Purification protocol 2. The cell pellet was resuspended in $1 \times$ Tris-EDTA-sucrose (TES) buffer and incubated for $1 \mathrm{hr}$. The lysate was then diluted by three using $0.25 \times$ TES buffer and incubated for $45 \mathrm{~min}$. Cellular fragments were removed by centrifugation $\left(9200 \times \mathrm{g}\right.$ for $1.5 \mathrm{~h}$ at $\left.4^{\circ} \mathrm{C}\right)$. The supernatant was incubated overnight at $4^{\circ} \mathrm{C}$ under gentle agitation with Ni-NTA agarose beads in phosphate buffered saline (PBS) (sodium phosphate $50 \mathrm{mM}, \mathrm{NaCl} 150 \mathrm{mM}$, pH 7.4) complemented with $10 \mathrm{mM}$ imidazole. Beads were washed with $\sim 20$ volumes of PBS containing $20 \mathrm{mM}$ imidazole, and with $\sim 5$ volumes of PBS complemented with $40 \mathrm{mM}$ imidazole. His-tagged proteins were eluted with $\sim 5$ volumes of PBS complemented with $0.5 \mathrm{M}$ imidazole. The buffer was exchanged to PBS (50 mM phosphate, $150 \mathrm{mM} \mathrm{NaCl}, \mathrm{pH} 7.4$ ) using PD-10 desalting columns. In both protocols, purity of the proteins was evaluated using SDS-PAGE electrophoresis stained with Coomassie blue.

Spectroscopic characterization. Steady state UV-Vis absorption spectra were recorded using a Cary 300 UV-Vis spectrometer (Agilent Technologies), equipped with a Versa20 Peltier-based temperature-controlled cuvette chamber (Quantum Northwest) and fluorescence data were recorded using a LPS 220 spectrofluorometer (PTI, Monmouth Junction, NJ), equipped with a TLC50TM Legacy/PTI Peltier-based temperature-controlled cuvette chamber (Quantum Northwest). Fluorescence quantum yield were determined as previously described using FAST:HBR-3,5DOM as reference ${ }^{16}$. 
Binding affinity. Thermodynamic dissociation constants were determined as previously described $^{12}$ using a Spark $10 \mathrm{M}$ plate reader (Tecan) and Prism 6 to fit the data with a one-site specific binding model.

\section{Molecular biology}

The plasmids pAG153 encoding FKBP-CFAST11 (= FKBP-C-frFAST) was previously described $^{20}$. The plasmids pAG29, pAG28, CV2326 encoding EGFP, Lyn11-EGFP, Mito-mCherry were previously described ${ }^{13,26}$. The plasmids pAG504, pAG505, pAG506 allowing the mammalian expression of frFAST, H2B-frFAST, mito-frFAST were obtained by replacing the sequence coding for FAST in the previously described ${ }^{12}$ pAG104, pAG109, pAG156 with that coding for frFAST using standard molecular biology techniques. The plasmid pAG324 allowing the mammalian expression of H2B-mCherry was obtained by replacing the sequence coding for dronpa2 in the previously described pAG123 with that coding for mCherry. The plasmid pAG502 allowing the mammalian expression of MAP4-frFAST was obtained by replacing the sequence coding for $\mathrm{H} 2 \mathrm{~B}$ in pAG505 with that coding for MAP4 using standard molecular biology techniques. The plasmids pAG499 and pAG500 for the mammalian expression of FRB-N-frFAST and FKBP-N-frFAST were obtained by replacing the sequence coding for N-FAST in the previously described ${ }^{20}$ pAG148 and pAG149 with that coding for N-frFAST using Gibson assembly.

For expression in zebrafish, the plasmids \#1115, \#1117, \#1120 and \#1121 encoding H2B fusion to frFAST, EGFP, iRFP713 and smURFP respectively were obtained by substituting mCherry downstream $\mathrm{H} 2 \mathrm{~B}$ in the $\mathrm{pT} 2 \mathrm{iC} 6$ vector (a derivative of $\mathrm{pCS} 2$ ) 
previously described $^{27}$. mRNA were synthesized with a mMessage mMachine ${ }^{\mathrm{TM}} \mathrm{SP} 6$ transcription kit (Thermofisher) according to manufacturer instructions. Plasmid \#1129 used to obtain transgenic fish line expressing LifeAct-frFAST fusion under the control of the cmlc2 enhancer ${ }^{28}$ was constructed using the NEBuilder assembly kit (New England BioLabs) in the pT22i vector for transgenesis previously used ${ }^{29}$.

For expression of H2B-frFAST in the chick neural tube, the CMV promoter in pAG505 was converted to a CAGGS promoter 22 by replacing the Ndel/Bglll fragment with a Ndel/Bglll CAGGS fragment from pCAGGS-MCS2 (X. Morin, unpublished). pCAGGSH2B-miRFP670nano was obtained by converting the CMV promoter in pH2BmiRFP670nano (https://www.addgene.org/127438/) to a CAGGS promoter by replacing the Ndel/Kpnl fragment with a Ndel/Kpnl fragment from pCAGGS-MCS2. pCAGGSmKO2-Farn was a kind gift of Fumio Matsuzaki.

Construction details, complete sequences and plasmids are available upon request.

\section{Experiments in mammalian cells}

General. HeLa cells were cultured in Modified Eagle Medium (MEM) supplemented with $0.9 \%$ MEM non-essential amino acids, $0.9 \%$ sodium pyruvate, and $9 \%$ (vol/vol) fetal calf serum (FCS), at $37^{\circ} \mathrm{C}$ in a $5 \% \mathrm{CO}_{2}$ atmosphere. U2OS cells were cultured in McCoy's 5A medium supplemented with phenol red and 10\% (vol/vol) FCS. HEK293T cells were cultured in Dulbecco's Modified Eagle Medium (DMEM) supplemented with phenol red, Glutamax I, and 10\% (vol/vol) FCS. For imaging, cells were seeded in $\mu$ Dish IBIDI (Biovalley) coated with poly-L-lysine. Cells were transiently transfected using Genejuice (Merck) according to the manufacturer's protocol for 24 hours prior to 
imaging. The cells were washed with PBS, and treated with DMEM media (without serum and phenol red) containing the fluorogens at the indicated concentration. The cells were imaged directly without washing.

Cell viability assay. HeLa cells were treated with DMEM media containing HPAR-3OM at the indicated concentrations for the indicated times. The cell viability was evaluated by fluorescence microscopy using the LIVE/DEAD® viability/cytotoxicity assay kit (Molecular Probes, Life Technologies) following the manufacturer's protocol.

Sensing of protein-protein interactions. To monitor the association of the FRB-FKBP homodimer, rapamycin was added to HEK 293T cells co-expressing FRB-N-frFAST and FKBP-C-frFAST to a final concentration of $500 \mathrm{nM}$. To measure the dissociation of the FKBP-FKBP homodimer, HEK293T cells co-expressing FKBP-N-frFAST and FKBP-CfrFAST were first pre-incubated with 100 nM AP1510 for 2 hours then rapamycin was added to a final concentration of $1.1 \mu \mathrm{M}$ for inducing the dissociation. Rapamycin was purchased from Sigma Aldrich and dissolved in DMSO to a concentration of $3 \mathrm{mM}$. AP1510 was purchased from Clontech and dissolved in ethanol to a concentration of $0.5 \mathrm{mM}$.

Microscopy. The confocal micrographs of mammalian cells were acquired on a Zeiss LSM 710 Laser Scanning Microscope equipped with a Plan Apochromat $63 \times / 1.4$ NA oil immersion objective. ZEN software was used to collect the data. The images were analyzed with Fiji (Image J).

\section{Experiments in zebrafish embryos and larvae}

Evaluation of fluorogen toxicity. Embryos were incubated in $5 \mu \mathrm{M}$ HPAR-3OM during 
$1 \mathrm{~h}$ at $50 \%$ epiboly or overnight from $50 \%$ epiboly to $24 \mathrm{hpf}$. Embryos with no defect, axis defects, or dead were scored at $48 \mathrm{hpf}$ by brightfield microscopy.

Evaluation of expression toxicity. mRNA coding for H2B-EGFP (20 ng/ $\mu$ l) and either H2B-frFAST (80 ng/ $\mu \mathrm{l})$ or H2B-smURFP (80 $\mathrm{ng} / \mu \mathrm{l})$ were co-injected at the one-cell stage. Embryos with no defect, axis defects, or dead were scored at 24 hpf by brightfield microscopy.

Brightness characterization. mRNA coding for H2B-EGFP (20 ng/ $\mu$ l) and either H2BfrFAST $(80 \mathrm{ng} / \mu \mathrm{l})$ or H2B-iRFP713 (80 $\mathrm{ng} / \mu \mathrm{l})$ were co-injected at the one-cell stage. frFAST expressing embryos were incubated with $5 \mu \mathrm{M}$ HPAR-3OM for 30 min prior imaging. Fluorescence of frFAST and iRFP713 was quantified by confocal microscopy and normalized to the fluorescence of EGFP.

Transgenic line. Plasmid \#1129 (10 ng/ $\mu \mathrm{L})$ was co-injected with mRNA coding for Tol2 transposase $(20 \mathrm{ng} / \mu \mathrm{L})$ and fish were screened for germline transmission.

Microscopy. Imaging of embryos and larvae embeded in low-melting agarose (0.8\%) was performed with a CSU-W1 Yokogawa spinning disk coupled to a Zeiss Axio observer Z1 inverted microscope equipped with a sCMOS Hamamatsu camera and a 25× (Zeiss 0.8 Imm WD: $0.19 \mathrm{~mm}$ ) oil objective. For frFAST and iRFP713 imaging, 561 $\mathrm{nm}$ and $642 \mathrm{~nm}$ lasers, and a 655 longpass filter were used. EGFP imaging was performed using a $491 \mathrm{~nm}$ laser and a $522 / 50$ bandpass filter.

\section{Experiments in chicken embryos}

JA57 chicken fertilized eggs were provided by EARL Morizeau (8 rue du Moulin, 28190 Dangers, France) and incubated at $38^{\circ} \mathrm{C}$ in a Sanyo MIR-253 incubator. Embryos used 
in this study were between E2 $(\mathrm{HH} 14)$ and E3 $(\mathrm{HH} 14+24 \mathrm{~h})$. The sex of the embryos was not determined.

Electroporation in the chick neural tube was performed at embryonic day 2 (E2, $\mathrm{HH}$ stage 14), by applying five pulses of $50 \mathrm{~ms}$ at $25 \mathrm{~V}$ with $100 \mathrm{~ms}$ in between, using a square-wave electroporator (Nepa Gene, CUY21SC) and a pair of 5-mm gold-plated electrodes (BTX Genetrode model 512) separated by a 4-mm interval. The DNA solution was injected directly into the lumen of the neural tube via glass capillaries. Bilateral electroporation was achieved by switching the electrodes polarity and repeating the procedure after $45 \mathrm{~min}$. All DNA constructs were used at $0.5 \mu \mathrm{g} / \mu \mathrm{l}$ each. En-face culture of the embryonic neuroepithelium was performed at E3 (24 h after electroporation). After extraction from the egg and removal of extraembryonic membranes in $\mathrm{PBS}$, embryos were transferred to $37^{\circ} \mathrm{C} \mathrm{F12} \mathrm{medium} \mathrm{and} \mathrm{pinned} \mathrm{down}$ with dissection needles at the level of the hindbrain and hindlimbs in a $35 \mathrm{~mm}$ Sylgard dissection dish. A dissection needle was used to separate the neural tube from the somites from hindbrain to caudal end on both sides of the embryo, and the roof-plate was then slit with the needle. The neural tube and notochord were then "peeled off" from the remaining tissues and equilibrated 5 min in 1\% low meting point agarose/F12 medium at $38^{\circ} \mathrm{C}$. The tissue was then transferred in a drop of agarose medium to a glass-bottom culture dish (MatTek, P35G-0-14-C) and excess medium was removed so that the neural tube would flatten with its apical surface facing the bottom of the dish, in an inverted open book conformation. After $30 \mathrm{~s}$ of polymerization on ice, an extra layer of agarose medium was added to cover the whole tissue and hardened on ice for $1 \mathrm{~min}$. $2 \mathrm{~mL}$ of $37^{\circ} \mathrm{C}$ culture medium was added (F12/Penicillin Streptomycin/Sodium pyruvate) 
and the culture dish was transferred to the $37^{\circ} \mathrm{C}$ chamber of a spinning disk confocal microscope. To image frFAST, $0.5 \mathrm{ml}$ of culture medium with $50 \mu \mathrm{M}$ HPAR-3OM (1.25 $\mu \mathrm{l}$ of a $20 \mathrm{mM}$ stock in $500 \mu \mathrm{l}$ medium) was added to the dish for a final concentration of $10 \mu \mathrm{M}$.

Live imaging was performed on an inverted microscope (Nikon Ti Eclipse) equipped with a heating enclosure (DigitalPixel, UK), a spinning disk confocal head (Yokogawa CSU-W1), and an sCMOS Camera (Orca Flash4LT, Hamamatsu) driven by MicroManager software ${ }^{30}$. Image stacks were obtained at 1 min intervals either with a 10× objective (CFI Plan APO LBDA, NA 0.45, Nikon; z-step $=4 \mu \mathrm{m}$; Figure 4c, Movie S1) or a 100 $\times$ oil immersion objective (APO VC, NA 1.4, Nikon; z-step = $1 \mu \mathrm{m}$; Figure $4 f-h)$. 
$\mathbf{a}$
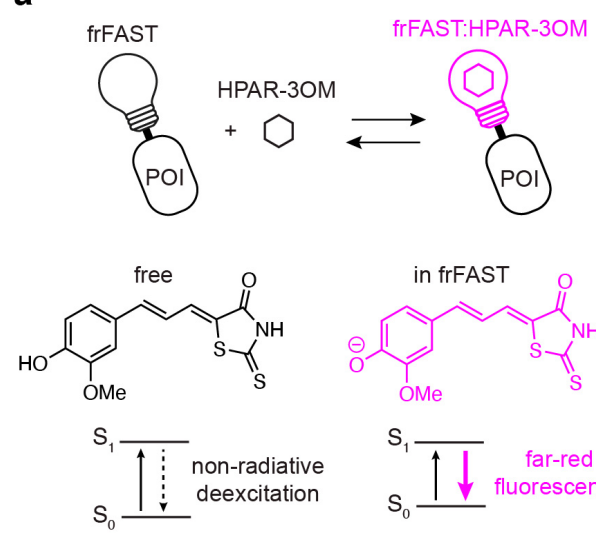

b

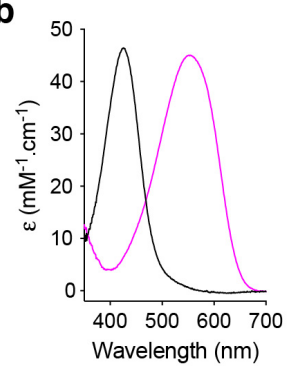

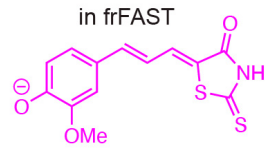

$\mathrm{S}_{1}$
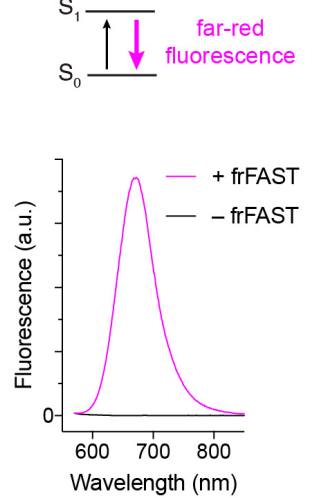

C
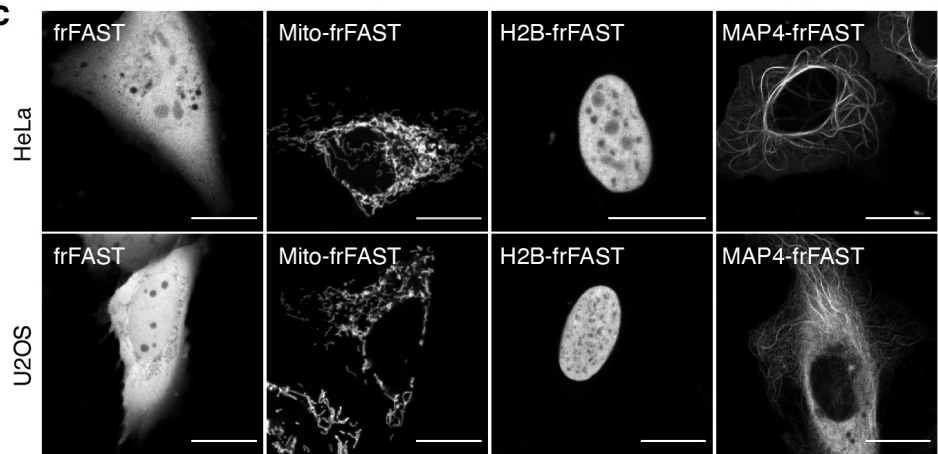

d
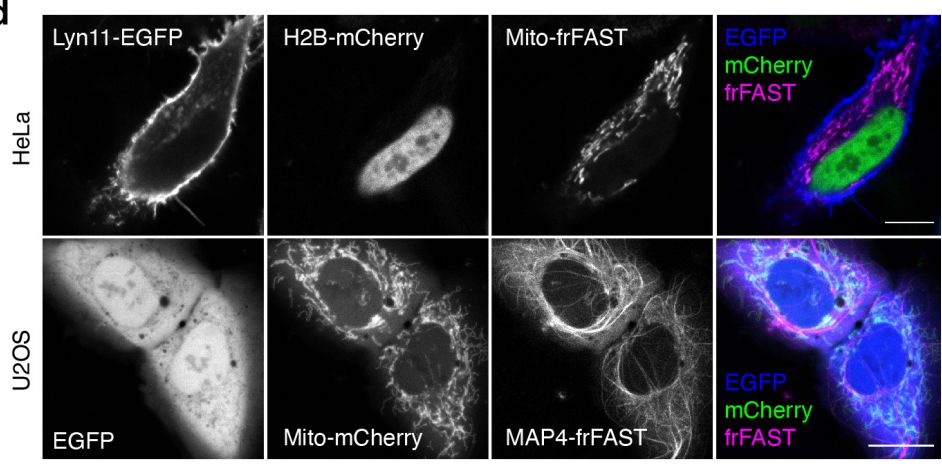

Figure 1. frFAST forms a far-red fluorescent assembly with HPAR-3OM and enables to encode far-red fluorescence in cells. (a) frFAST principle and structure of HPAR-3OM (POI: protein of interest). (b) Absorption and emission spectra of the HPAR-3OM in absence (black) or presence (magenta) of frFAST. HPAR-3OM and frFAST concentrations were $2 \mu \mathrm{M}$ and $40 \mu \mathrm{M}$, respectively, in pH 7.4 PBS (50 mM sodium phosphate, $150 \mathrm{mM} \mathrm{NaCl}$ ). Spectra were recorded at $25^{\circ} \mathrm{C}$. (c) Confocal micrographs of live HeLa and U2OS cells expressing various frFAST fusions. Cells were incubated with $10 \mu \mathrm{M}$ HPAR-3OM for $15-30$ seconds and then directly imaged using the following settings: Ex/Em 633/638-797 nm. (d) Confocal micrographs of live HeLa and U2OS cells co-expressing various EGFP, mCherry and frFAST fusions. Cells were incubated with $10 \mu \mathrm{M}$ HPAR-3OM for $15-30$ seconds and then directly imaged using the following settings: EGFP Ex/Em 488/493-599 nm, mCherry Ex/Em 543/560598, frFAST Ex/Em 633/650-797 nm. (c,d) Mito: Mitochondrial targeting sequence from the subunit VIII of human cytochrome $c$ oxidase, H2B: Histone 2B, MAP4: microtubuleassociated protein 4, Lyn11: membrane localization signal. Scale bars $10 \mu \mathrm{m}$. 

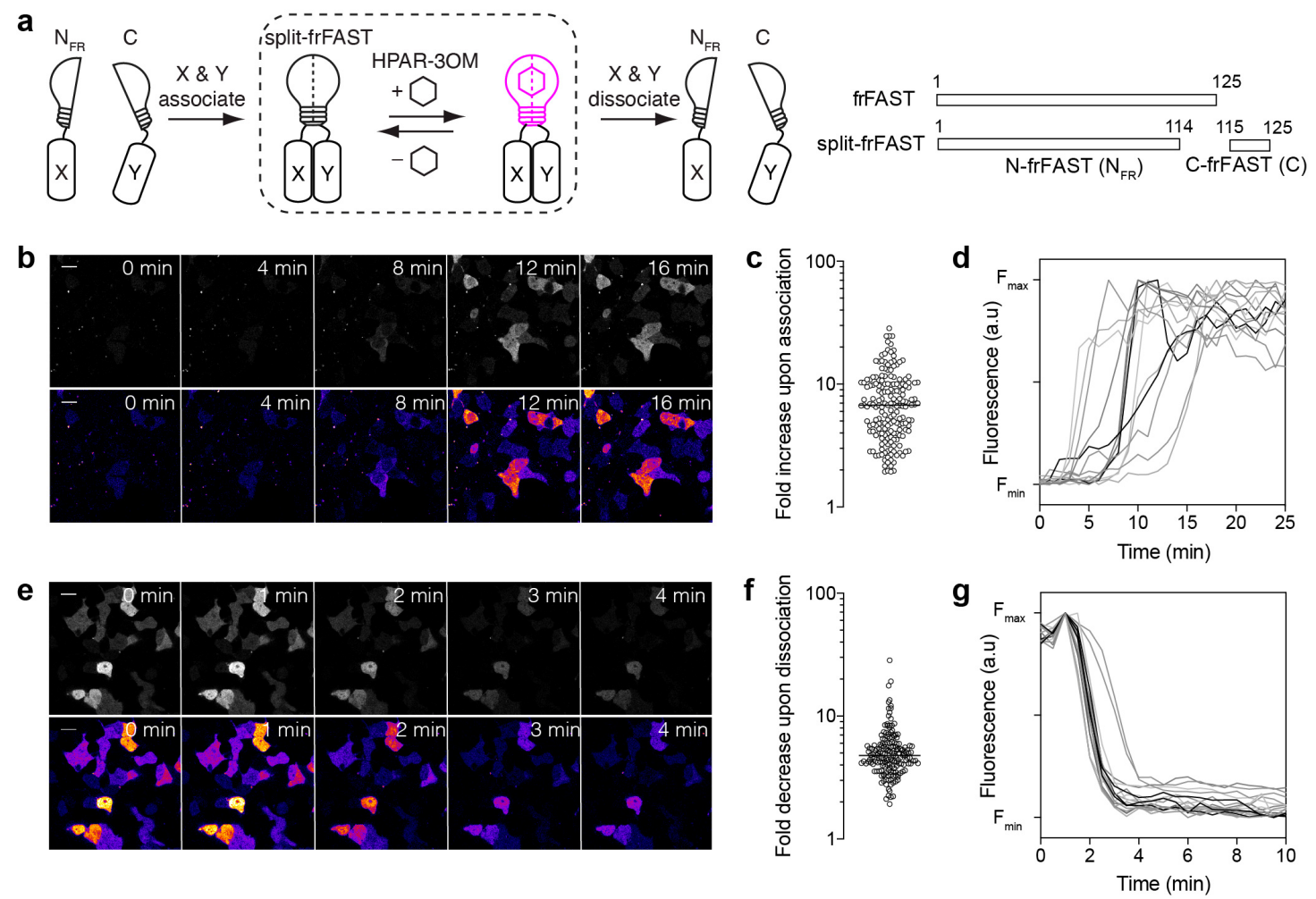

Figure 2. Design of a far-red fluorescent reporter for the detection of dynamic protein-protein interactions. (a) Principle and design of split-frFAST. (b-d) HEK293T cells co-expressing FK506-binding protein (FKBP)-C-frFAST and FKBP-rapamycinbinding domain of mammalian target of rapamycin (FRB)-N-frFAST were labeled with $10 \mu \mathrm{M}$ HPAR-3OM, and imaged before and after addition of $500 \mathrm{nM}$ rapamycin. (b) Representative time-lapse. Scale bar $20 \mu \mathrm{m}$. (c) Fluorescence fold increase upon FKBP-FRB association, $n=185$ cells from three experiments. The line indicates the median. (d) Temporal evolution of the fluorescence intensity after rapamycin addition in HPAR-3OM-treated cells co-expressing FRB-N-frFAST and FKBP-C-frFAST ( $n=14$ cells). (e-g) HEK293T cells co-expressing FKBP-N-frFAST and FKBP-C-frFAST were treated with $100 \mathrm{nM}$ AP1510 and labeled with $10 \mu \mathrm{M}$ HPAR-3OM. Cells were then imaged before and after the addition of $1.1 \mu \mathrm{M}$ rapamycin. (e) Representative timelapse. Scale bar $20 \mu \mathrm{m}$. (f) Fluorescence fold decrease upon FKBP-FKBP dissociation, $n=170$ cells, from three experiments. The line indicates the median. (g) Temporal evolution of the fluorescence intensity after rapamycin addition in AP1510-treated cells co-expressing FKBP-N-frFAST and FKBP-C-frFAST ( $n=18$ cells). 
a

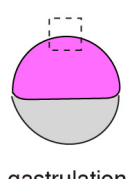

gastrulation b
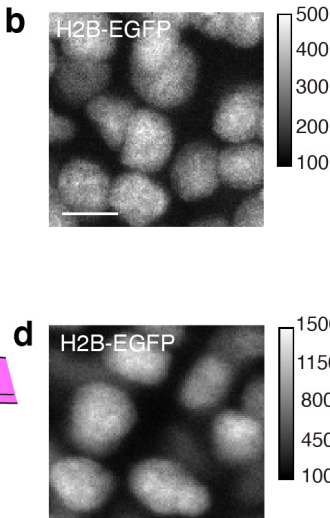

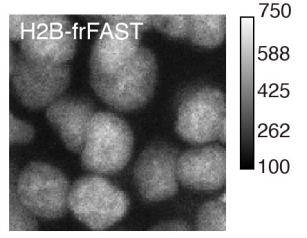

c
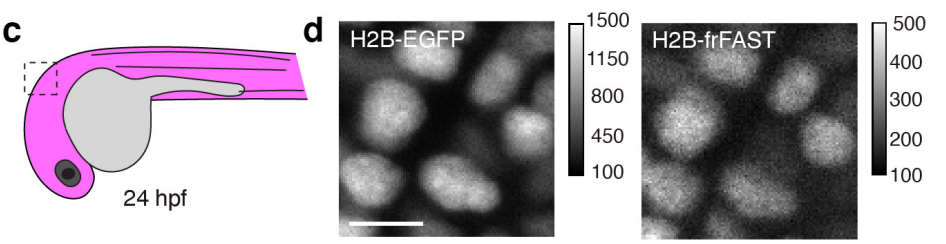
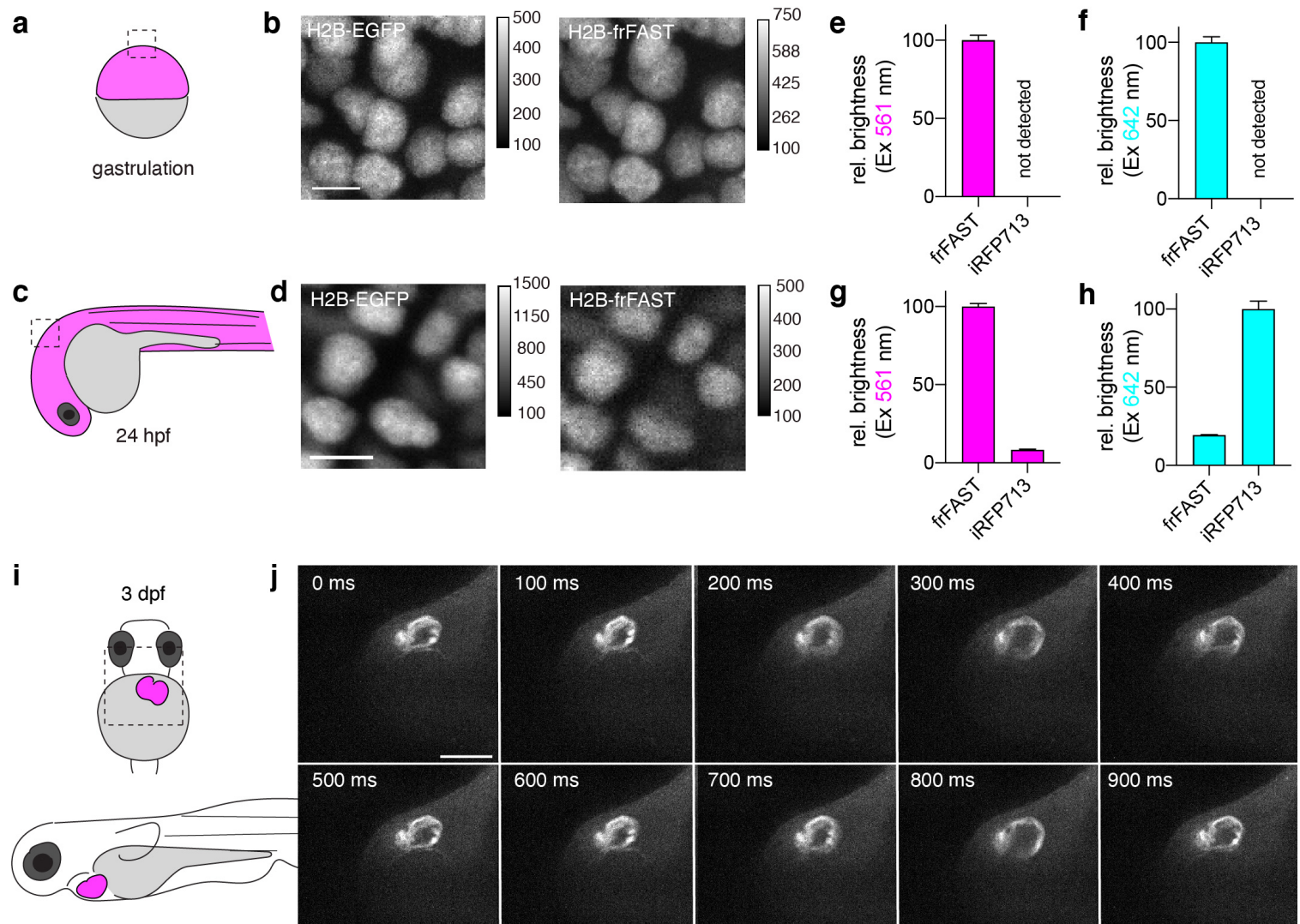

$\operatorname{Tg}(\mathrm{cm} / \mathrm{c} 2:$ LifeAct-frFAST $)$
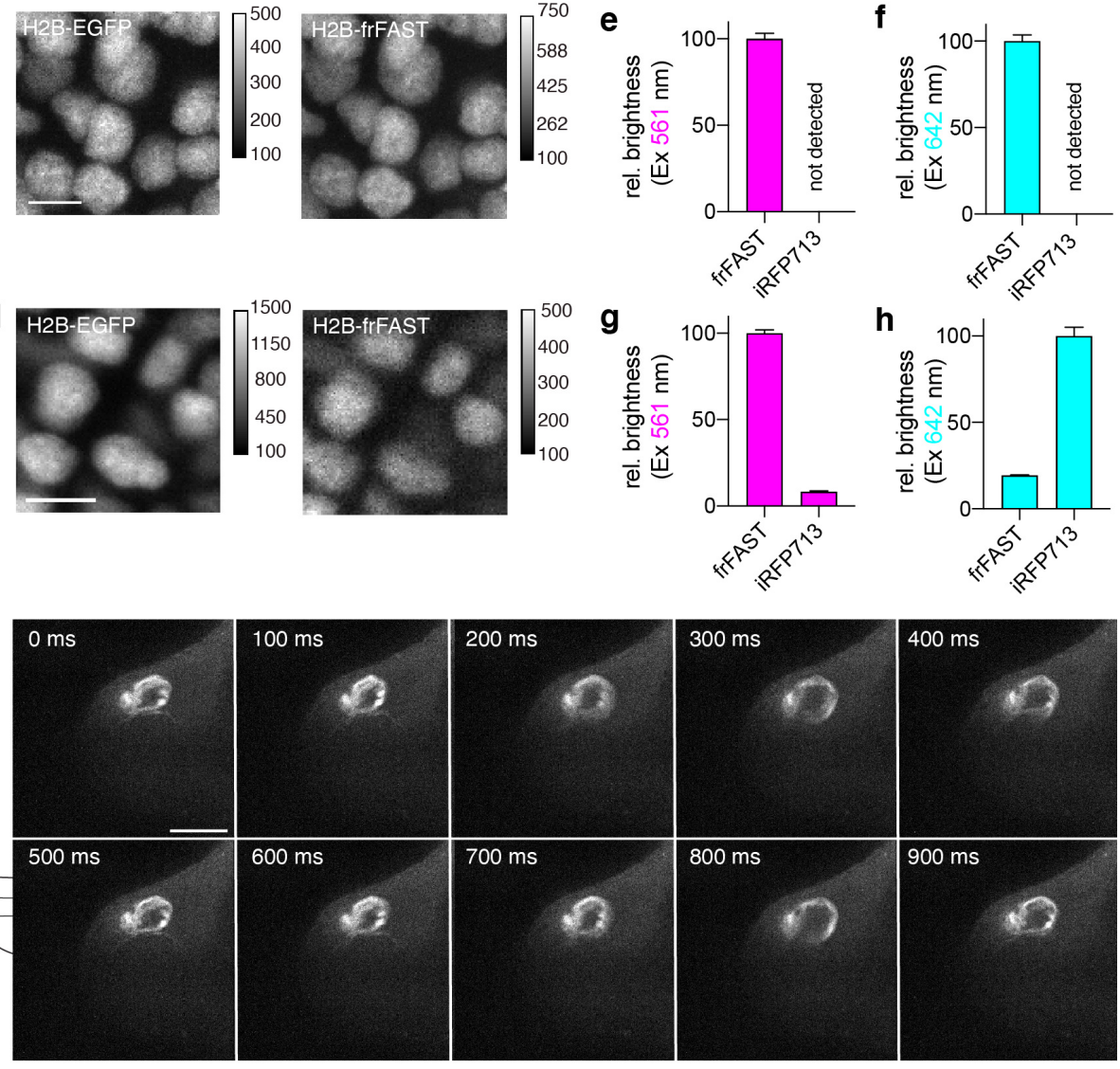

Figure 3. frFAST enables to encode far-red fluorescence in zebrafish embryos and larvae. (a-d) mRNA encoding H2B-EGFP and H2B-frFAST were micro-injected in zebrafish embryo at the one-cell stage. The confocal micrographs shows few cells in zebrafish embryos during gastrulation (a,b) and at $24 \mathrm{hpf}(\mathbf{c}, \mathbf{d})$. Embryos were incubated with $5 \mu \mathrm{M}$ HPAR-3OM for 30 min prior imaging. Micrographs were recorded with a spinning-disk confocal microscope with the following settings: EGFP Ex $491 \mathrm{~nm} /$ Em 497-547 nm, frFAST Ex 561 nm / Em LP 655 nm. Scale bars $10 \mu \mathrm{m}$. (e-h) Effective brightness of frFAST and iRFP713 during gastrulation (e,f) and at $24 \mathrm{hpf}(\mathbf{g}, \mathbf{h})$ exciting at $561 \mathrm{~nm}(\mathbf{e}, \mathbf{g})$ or $642 \mathrm{~nm}(\mathbf{f}, \mathbf{h})$. frFAST and iRFP713 were expressed in zebrafish embryo as fusion to $\mathrm{H} 2 \mathrm{~B}$, and fluorescence was quantified by confocal microscopy. The relative brightness were determined after normalization to fluorescence of co-expressed H2B-EGFP. Brightness represents the mean \pm sem determined from $n>50$ cells. (i,j) Time-lapse imaging of a $3 \mathrm{dpf}$ transgenic zebrafish larva expressing LifeAct-frFAST in the myocardium (see also Supplementary Movie 1). The larva was incubated with 5 $\mu \mathrm{M}$ HPAR-3OM for $2 \mathrm{~h}$ prior imaging. Micrographs were recorded with a spinning-disk confocal microscope with the following settings: frFAST Ex $561 \mathrm{~nm} / \mathrm{Em}$ LP $655 \mathrm{~nm}$. Scale bar $100 \mu \mathrm{m}$. 
a

b

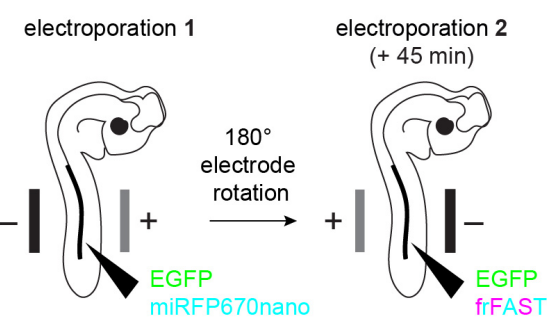

c
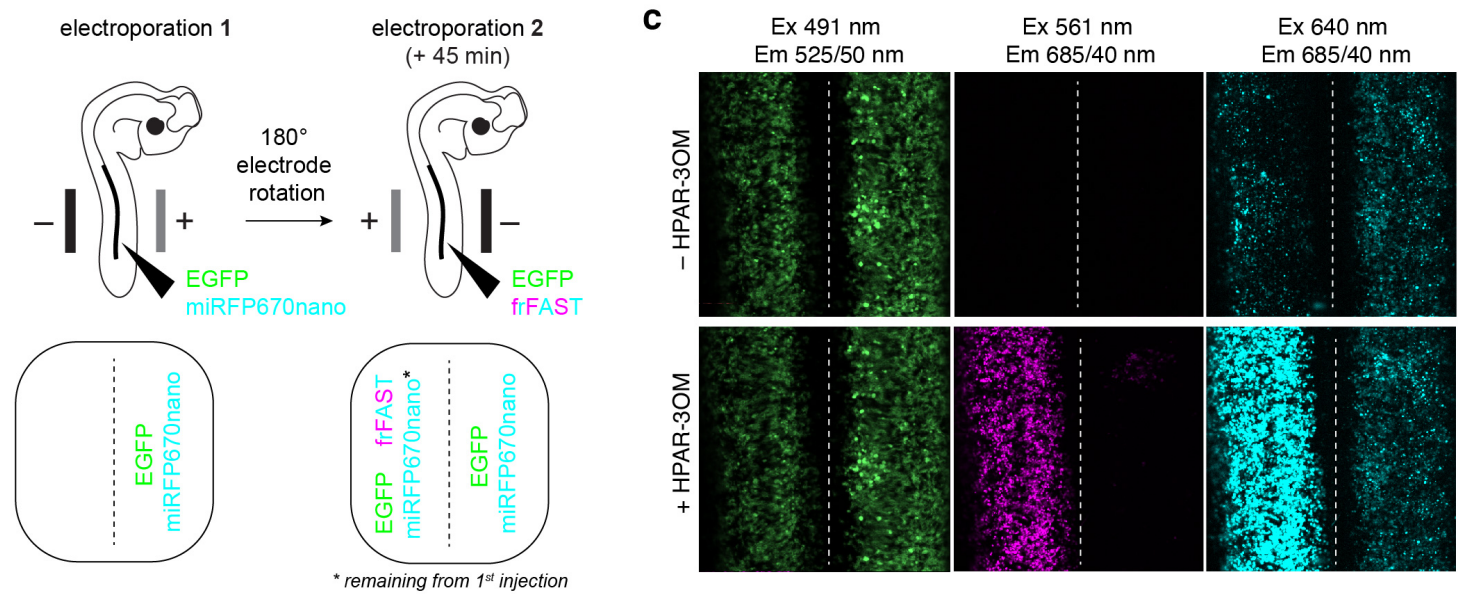

d

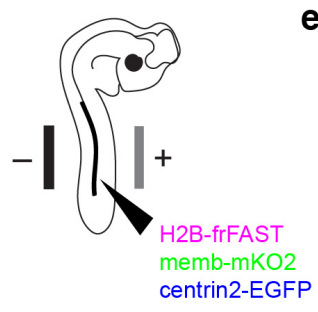

e

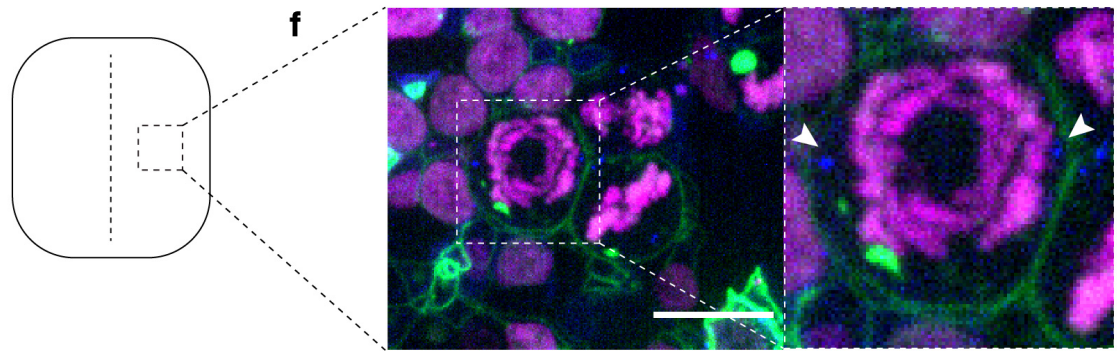

g

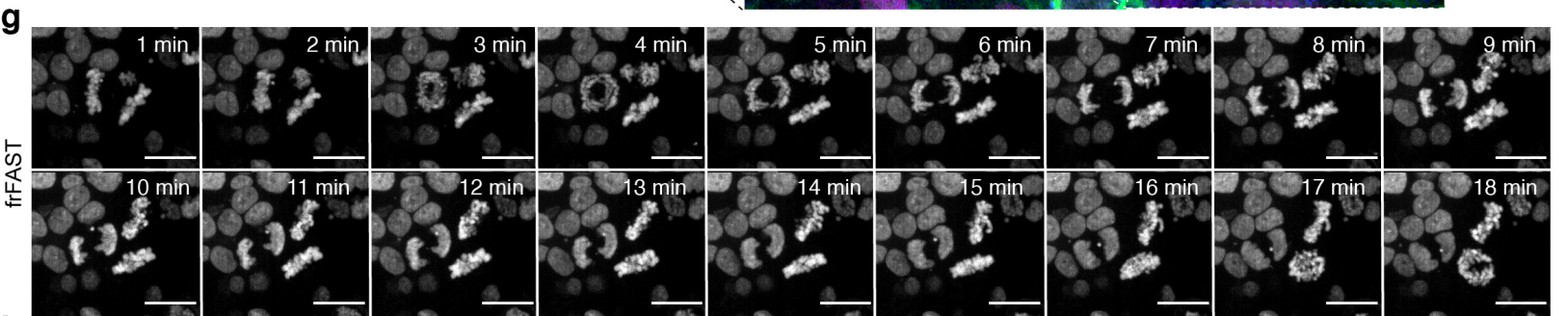

h

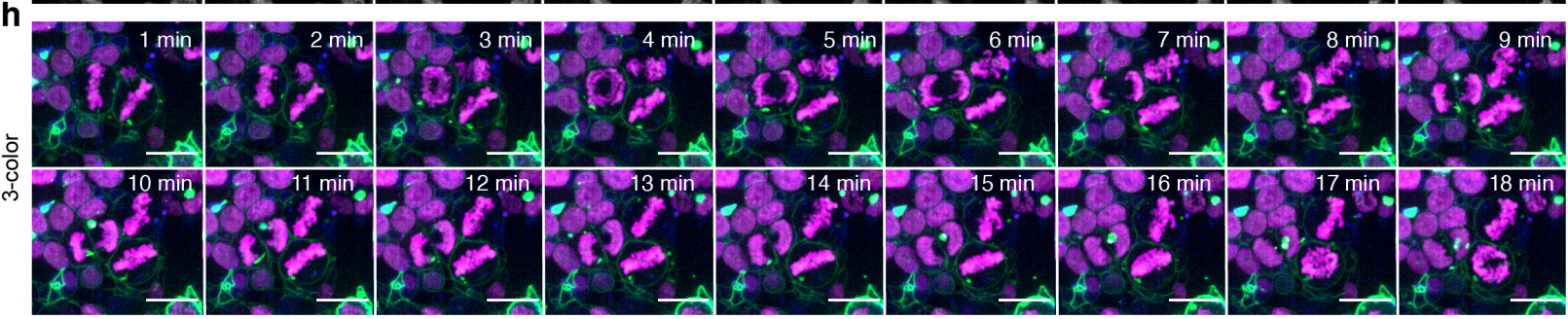

Figure 4. frFAST enables to encode far-red fluorescence in chicken embryos. (a-c) Plasmids encoding H2B-miRFP670nano and H2B-frFAST were each electroporated in one of each side of the neural tube in ovo at embryonic day 2 (E2, HH stage 13-14) (a). An EGFP reporter (fused to a membrane localization signal) was co-injected with each construct to monitor electroporation efficiency. $24 \mathrm{~h}$ later, embryos with homogeneous bilateral EGFP expression in the neural tube were dissected (b), and imaged in absence and in presence of $10 \mu \mathrm{M}$ HPAR-3OM using a spinning-disk confocal microscope (c) (see Supplementary Movie 2 for time-lapse imaging of the labeling). Note that before addition of HPAR-3OM, some miRFP670nano signal is detected on the left side of the embryo. This is caused by residual plasmid from the first injection 
remaining in the neural tube at the time of the second injection. (d-h) Multicolor imaging. Plasmids encoding H2B-frFAST, mKO2 (fused to a membrane localization signal) and Centrin2-EGFP were electroporated in the neural tube in ovo, at embryonic day 2 (E2, $\mathrm{HH}$ stage 13-14) (d,e). $24 \mathrm{~h}$ later, embryos were dissected, and imaged in presence of $10 \mu \mathrm{M}$ HPAR-3OM using a spinning-disk confocal microscope. (f) Three-color micrographs showing the correct localization of the three proteins (Centrin2-EGFP is indicated with white arrows). (g,h) Time-lapse showing cell division. Micrographs were recorded with the following settings: EGFP Ex $491 \mathrm{~nm} / \mathrm{Em}$ 525/50 nm, mKO2 Ex 561 nm / Em 617/73 nm, frFAST Ex 561 nm / Em 685/40 nm. Scale bar $10 \mu \mathrm{m}$. 


\section{REFERENCES}

1. Shu, X. et al. Mammalian Expression of Infrared Fluorescent Proteins Engineered from a Bacterial Phytochrome. Science 324, 804-807 (2009).

2. Filonov, G. S. et al. Bright and stable near-infrared fluorescent protein for in vivo imaging. Nat. Biotechnol. 29, 759-763 (2011).

3. Shcherbakova, D. M. \& Verkhusha, V. V. Near-infrared fluorescent proteins for multicolor in vivo imaging. Nat. Meth. 10, 751-754 (2013).

4. Shcherbakova, D. M. et al. Bright monomeric near-infrared fluorescent proteins as tags and biosensors for multiscale imaging. Nat. Commun. 7, 12405 (2016).

5. Rodriguez, E. A. et al. A far-red fluorescent protein evolved from a cyanobacterial phycobiliprotein. Nat. Meth. 13, 763-769 (2016).

6. Oliinyk, O. S., Shemetov, A. A., Pletnev, S., Shcherbakova, D. M. \& Verkhusha, V. V. Smallest near-infrared fluorescent protein evolved from cyanobacteriochrome as versatile tag for spectral multiplexing. Nat. Commun. 10, 279-13 (2019).

7. Shemetov, A. A., Oliinyk, O. S. \& Verkhusha, V. V. How to Increase Brightness of Near-Infrared Fluorescent Proteins in Mammalian Cells. Cell Chem. Biol. 24, 758766.e3 (2017).

8. Szent-Gyorgyi, C. et al. Fluorogen-activating single-chain antibodies for imaging cell surface proteins. Nat. Biotechnol. 26, 235-240 (2008).

9. Lukinavicius, G. et al. A near-infrared fluorophore for live-cell super-resolution microscopy of cellular proteins. Nat. Chem. 5, 132-139 (2013).

10. Lukinavicius, G. et al. Fluorogenic Probes for Multicolor Imaging in Living Cells. J. Am. Chem. Soc. 138, 9365-9368 (2016).

11. Grimm, J. B. et al. A general method to fine-tune fluorophores for live-cell and in vivo imaging. Nat. Meth. 14, 987-994 (2017).

12. Plamont, M.-A. et al. Small fluorescence-activating and absorption-shifting tag for tunable protein imaging in vivo. Proc. Natl. Acad. Sci. USA 113, 497-502 (2016).

13. Li, C. et al. Dynamic multicolor protein labeling in living cells. Chem. Sci. 8, 55985605 (2017).

14. Monmeyran, A. et al. The inducible chemical-genetic fluorescent marker FAST outperforms classical fluorescent proteins in the quantitative reporting of bacterial biofilm dynamics. Sci. Rep. 8, 10336 (2018).

15. Streett, H. E., Kalis, K. M. \& Papoutsakis, E. T. A Strongly Fluorescing Anaerobic Reporter and Protein-Tagging System for Clostridium Organisms Based on the Fluorescence-Activating and Absorption-Shifting Tag Protein (FAST). Appl. Environ. Microbiol. 85, 714 (2019).

16. Tebo, A. G., Pimenta, F. M., Zhang, Y. \& Gautier, A. Improved Chemical-Genetic Fluorescent Markers for Live Cell Microscopy. Biochemistry 57, 5648-5653 (2018).

17. Venkatachalapathy, M., Belapurkar, V., Jose, M., Gautier, A. \& Nair, D. Live cell super resolution imaging by radial fluctuations using fluorogen binding tags.

Nanoscale 124, 1607 (2019). 
18. Smith, E. M., Gautier, A. \& Puchner, E. M. Single-Molecule Localization Microscopy with the Fluorescence- Activating and Absorption-Shifting Tag (FAST) System. ACS Chem. Biol. 14, 1115-1120 (2019).

19. Tebo, A. G. et al. Circularly Permuted Fluorogenic Proteins for the Design of Modular Biosensors. ACS Chem. Biol. 13, 2392-2397 (2018).

20. Tebo, A. G. \& Gautier, A. A split fluorescent reporter with rapid and reversible complementation. Nat. Commun. 10, 2822-8 (2019).

21. Li, C. et al. Design and characterization of red fluorogenic push-pull chromophores holding great potential for bioimaging and biosensing. Org. Biomol. Chem. 14, 9253-9261 (2016).

22. Niwa, H., Yamamura, K. \& Miyazaki, J. Efficient selection for high-expression transfectants with a novel eukaryotic vector. Gene 108, 193-199 (1991).

23. Gibson, D. G. et al. Enzymatic assembly of DNA molecules up to several hundred kilobases. Nat. Meth. 6, 343-345 (2009).

24. Tebo, A. G. et al. Orthogonal fluorescent chemogenetic reporters for multicolor imaging. see bioRxiv.

25. Gietz, R. D. \& Schiestl, R. H. Large-scale high-efficiency yeast transformation using the LiAc/SS carrier DNA/PEG method. Nat. Protoc. 2, 38-41 (2007).

26. Querard, J. et al. Photoswitching kinetics and phase-sensitive detection add discriminative dimensions for selective fluorescence imaging. Angew. Chem. Int. Ed. 54, 2633-2637 (2015).

27. Rampon, C. et al. Control of brain patterning by Engrailed paracrine transfer: a new function of the Pbx interaction domain. Development 142, 1840-1849 (2015).

28. Huang, C.-J., Tu, C.-T., Hsiao, C.-D., Hsieh, F.-J. \& Tsai, H.-J. Germ-line transmission of a myocardium-specific GFP transgene reveals critical regulatory elements in the cardiac myosin light chain 2 promoter of zebrafish. Dev. Dyn. 228, 30-40 (2003).

29. Meda, F. et al. Nerves Control Redox Levels in Mature Tissues Through Schwann Cells and Hedgehog Signaling. Antioxid. Redox Signal. 24, 299-311 (2016).

30. Edelstein, A., Amodaj, N., Hoover, K., Vale, R. \& Stuurman, N. Computer Control of Microscopes Using $\mu$ Manager. Curr. Protoc. Mol. Biol. 92, 1-17 (2010). 